\title{
Impact of Plesiomonas Shigelloides Strain CNCTC 144/92 Lipopolysaccharide on DPPC Liposome Membranes: EPR Method
}

\author{
D. MAN ${ }^{a, *}$, B. PYTEL ${ }^{a}$ AND I. PISAREK ${ }^{b}$ \\ ${ }^{a}$ Institute of Physics, Opole University, Oleska 48, 45-052 Opole, Poland \\ ${ }^{b}$ Department of Land Protection, Opole University, 45-052 Opole, Oleska 22, Poland
}

\begin{abstract}
Membrane fluidity measurements were performed for synthetic DPPC liposomes sonicated in aqueous solution and doped by Plesiomonas shigelloides strain CNCTC 144/92 (serotype O74:H5) lipopolysaccharide (LPS) extracted from the phenol (LPS $\mathrm{PhOH})$ and water $\left(\mathrm{LPS}_{\mathrm{H}_{2} \mathrm{O}}\right)$ phases. Concentrations of LPS in relation to DPPC ranged from 0 to $1.4 \%$ (molar ratio). The EPR spin probe method was used to describe physicochemical properties of different regions of the lipid bilayer. Since TEMPO spin probe dissolves both in the hydrophobic region of the membrane and in an aquatic environment it is possible to determine the spectroscopic partition parameter $F$, indicating the changes that occur in the water-lipid interface. The 16-DOXYL probe distributed in the middle of the lipid bilayer makes it possible to obtain the rotational correlation time $\tau$ parameter, which provides information about fluidity changes in the liposome membrane. Here we report that increasing concentrations (mainly in the range of $0.4-0.8 \%$ ) of investigated $\mathrm{LPS} \mathrm{PhOH}_{\mathrm{H}}$ and $\mathrm{LPS}_{\mathrm{H}_{2} \mathrm{O}}$ significantly influence spectroscopic parameters $(F$ and $\tau)$. The surface area of the DPPC liposomes membranes was affected predominantly by $\operatorname{LPS}_{\mathrm{H}_{2} \mathrm{O}}$ while the lipid bilayer was most influenced by $\mathrm{LPS}_{\mathrm{PhOH}}$.
\end{abstract}

DOI: 10.12693/APhysPolA.132.77

PACS/topics: 87.16.Dg, 87.64.kh, 87.80.Lg

\section{Introduction}

Electron paramagnetic resonance (EPR) is a technique used to investigate membrane fluidity impacted by biologically active substances through the spin probe method [1-3]. Due to diamagnetic properties of biological structures the spin probe method is suitable for EPR spectroscopy [4-6]. The structure of the liposomes resembles biological membranes, therefore they are used as model membranes in many experiments [3, 7]. Sonication is used to obtain $200 \mathrm{~nm}$ in diameter monolayer liposomes. The advantage of liposomes in research is the regulation of their molecular composition, which in natural biological membranes is very difficult. The present study investigated the effect of LPS molecules, important from a medical point of view. LPS molecules were extracted from the bacteria Plesiomonas shigelloides by researchers at the Department of Immunochemistry, Hirszfeld Institute of Immunology and Experimental Therapy, PAS.

Plesiomonas shigelloides are Gram-negative bacteria which are commonly distributed in the environment. They are most often isolated from domestic animals (poultry and swine), shellfish and fresh water (rivers, lakes, groundwater). Infections are correlated strongly with water contamination and occur mainly in tropical environments [8]. Human P. shigelloides infections typically cause traveler's diarrhea which is the most predictable travel-related illness especially in countries with low sanitary standards $[9,10]$. It is also an opportunis-

*corresponding author; e-mail: dariusz.man@uni.opole.pl tic pathogen in immunocompromised hosts [11, 12] and neonates [13]. The lipopolysaccharide (LPS) is an integral part of the Gram-negative outer membrane. Smooth LPS (S-LPS) is composed of three main regions: Ospecific polysaccharide (O-PS), core oligosaccharide and lipid A [14]. O-PS is the water-soluble region built of many oligosaccharide repeating units and extends outside of the bacteria surface. Lipid A, which is the most hydrophobic LPS region, is anchored directly to the bacteria membrane and when it is exposed to the external environment (e.g. during bacterial lysis) it is responsible for inducing the toxic effect mediated by interactions with cells of the host immune system. To extract LPS from Gram-negative bacteria the hot phenol/water method [15] is used. S-LPS is usually obtained from the water phase $\left(\mathrm{LPS}_{\mathrm{H}_{2} \mathrm{O}}\right)$. LPS of the $P$. shigelloides strain CNCTC 144/92 (serotype O74:H5) which is a S-LPS obtained with the hot phenol/water method unexpectedly showed higher yields from the phenol phase [16]. This unusual phenomenon was also reported for S-LPS from Yersinia spp., Azorhizobium and Acinetobacter [17-19]. Most of the S-type enterobacterial lipopolysaccharides are extracted from the water phase which indicates unusual physicochemical properties of the LPS P. shigelloides strain CNCTC 144/92. Here we present the results of the P. shigelloides strain CNCTC 144/92 LPS obtained from the phenol and water phases $\left(\mathrm{LPS}_{P h O H}\right.$ and $\mathrm{LPS}_{\mathrm{H}_{2} \mathrm{O}}$ ) and its concentration-dependent impact on the dynamic properties of sonicated dipalmitoilphosphatidylcholine (DPPC) liposome membranes. A lipid bilayer composed of DPPC at room temperature is in the gel phase (main phase transition recorded at approximately $41^{\circ} \mathrm{C}$ ) and is well suited to analyze the ef- 
fects of compounds that influence membrane fluidity. Liposomes composed of lecithin are diamagnetics therefore spin probes must be used for EPR. Spin probe 16DOXYL is a derivative of a fatty acid and locates in the membrane like phospholipid molecules. Therefore 16-DOXYL is commonly used in membrane EPR experiments. The TEMPO spin probe dissolves both in the hydrophobic region of the membrane and an aquatic environment. Therefore it can be used to determine the changes that occur in water-lipid interface.

\section{Materials and methods}

\subsection{Spin labels}

Two different spin labels (Fig. 1) (purchased from Sigma-Aldrich) were used in EPR tests. The first was a 2,2,6,6-tetramethylpiperidine-1-oxyl (TEMPO), empirical formula (Hill Notation) $\mathrm{C}_{9} \mathrm{H}_{18} \mathrm{NO}$, molecular weight 156.25 . a<smiles>CC1(C)CCCC(C)(C)N1[O]</smiles>

b

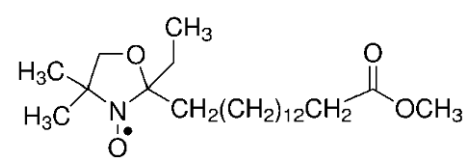

Fig. 1. The structures of spin labels: (a) TEMPO, (b) 16-DOXYL stearic acid methyl ester.

The second was a 2-ethyl-2-(15-methoxy-15oxopentadecyl)-4,4-dimethyl-3-oxazolidinyloxy (16DOXYL), empirical formula (Hill Notation) $\mathrm{C}_{23} \mathrm{H}_{44} \mathrm{NO}_{4}$, molecular weight 398.60 .

\subsection{LPS preparation}

LPS of P. shigelloides strain CNCTC 144/92 (serotype O74:H5) extracted from the phenol ( $\left.\mathrm{LPS}_{\mathrm{PhOH}}\right)$ and water $\left(\right.$ LPS $_{\mathrm{H}_{2} \mathrm{O}}$ ) phases was kindly provided by Prof. Czesław Lugowski from the Department of Immunochemistry, Ludwik Hirszfeld Institute of Immunology and Experimental Therapy, Polish Academy of Sciences, Wrocław, Poland.

\subsection{EPR method and liposome testing}

Liposomes were obtained from synthetic dipalmitoilphosphatidylcholine (DPPC) lecithin in distilled water, by sonication (ultrasonic disintegrator TECHPAN UD-20). The procedure included alternating cycles of $20 \mathrm{~s}$ of sonication followed by $30 \mathrm{~s}$ of cooling. The total preparation time was $2 \mathrm{~min}$ for each $1.0 \mathrm{ml}$ sample. The concentration of DPPC in each sample was fixed at $40 \mathrm{mM}$, whereas that of the spin label was $0.2 \%$ relative to the lecithin (i.e., molar ratio to lipid is 0.002). An EPR spectrometer MX-201R (TU Wrocław, Poland) was used in the tests under the following operating condition: the amplitude was $0.16 \mathrm{mT}$. In all experiments, the scanning range and the sweep speed of the EPR spectrometer were $5 \mathrm{mT}(50 \mathrm{G})$ and $64 \mathrm{~s}$, respectively, and the results are an average of three to four tests for an individual sample. EPR studies were carried out by using the same spin probe technique reported in our previous work [20].
The tested dopants (dissolved in DMF) were added to a suspension of previously formed liposomes, and their concentration was gradually increased from $0 \%$ to $1.4 \%$ (molar) relative to the lecithin. Measurements were performed at $24^{\circ} \mathrm{C}$, and the total time of a single test did not exceed $100 \mathrm{~min}$. Two diverse spin probes sensing different parts of the membrane were used. The TEMPO spin label penetrates to the interface region of the lipid bilayer and dissolves in both the hydrophobic part of the membrane and in the water medium. From the EPR spectra of this spin probe, the spectroscopic partition parameter $F=\frac{H}{H+P}$, reflecting the probe distribution between the membrane and its ambient, was determined. The value of $F$ is expressed as a ratio of amplitudes corresponding to the low-field line $H$ related to the lipid medium and the high-field line $P$ referred to as the water ambient (Fig. 2a). It is also related to the fluidity of the membrane's surface layer [21]. The 16-DOXYL spin label locates itself in the central region of the lipid bilayer. From the spectra of this probe, the spectroscopic parameter $\tau$ (rotational correlation time) was determined. The value of $\tau$ depends among other factors on the membrane degree of fluidity. The greater it is the more rigid (better organized) is the ambient in which the spin label has been placed [22] (Fig. 2b). A relative error determined in the measurements of $F$ and $\tau$ parameters was $2 \%$ and $3 \%$, respectively.

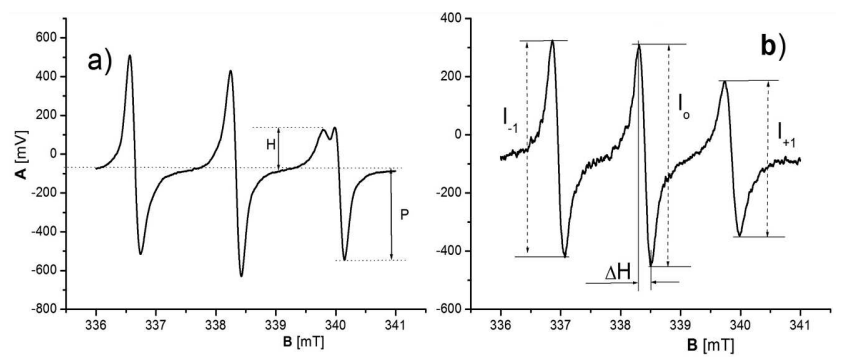

Fig. 2. ESR spectra provided by the TEMPO (a) and 16-DOXYL spin labels (b).

\section{Results and discussion}

The EPR spin probe method was used to determine dynamic properties (parameters $F$ and $\tau$ ) of the liposome membranes. Spin probes were selected depending on their location in different regions of the cross-section of the membrane (TEMPO, 16-DOXYL). Obtained results are presented in Fig. $3(F=f(c), \tau=f(c))$. Partition parameter $F$ formula (1) and $\tau$ parameter formula (2) are presented below. The formula defines the parameter $F$ - partition parameter (TEMPO spin labels):

$$
F=\frac{H}{H+P},
$$

$H$ - amplitude of low-field line of probe in lipid surrounding, $P$ - amplitude of high-field line of probe in $\mathrm{H}_{2} \mathrm{O}$ environment. An increase in the value of the $F$ parameter is connected with a higher concentration of probes dissolved in a lipid environment - an increase in 


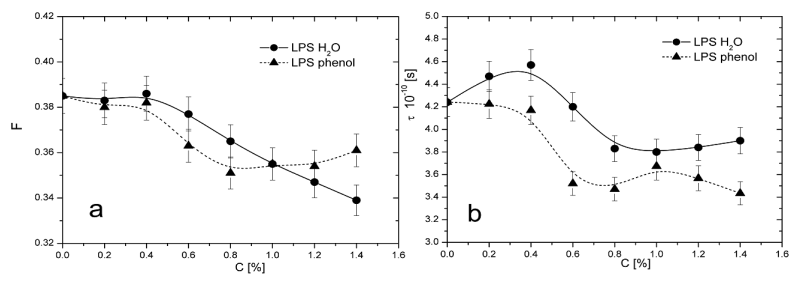

Fig. 3. Effect of the dopant concentration on the behavior of the spin labels within the DPPC liposome membrane, based on the measured EPR spectra: (a) changes in the partition parameter $F$ of the TEMPO, and (b) changes in the 16-DOXYL rotational correlation time $\tau$.

membrane fluidity in the surface layer and the center of the lipid bilayer.

The formula defines the parameter $\tau$ - rotation correlation time (16-DOXYL spin labels):

$$
\tau=5.95 \times 10^{-10} \Delta H_{0}\left(\sqrt{\frac{I_{0}}{I_{+1}}}+\sqrt{\frac{I_{0}}{I_{-1}}}-2\right)[\mathrm{s}],
$$

where $\Delta H_{0}$ - width of the centre line of the EPR spectrum of the spin probe, $I_{0}$ - amplitude of the centre line, $I_{+1}$ - amplitude of the high-field line, $I_{-1}$ - amplitude of the low-field line.

Based on the spectrum obtained by the 16-DOXYL probe, the spectroscopic parameter $\tau$ - rotation correlation time - was determined. The value of this parameter also depends on the degree of fluidity of the environment. The higher it is, the stiffer (the more ordered) is the environment in which the probe is immersed. In an isotropic environment parameter $\tau$ is a rotational correlation time of the probe. Data from Fig. 3 suggest that with increase of concentrations of LPS doped into a membrane, the spectroscopic parameters of the spin probes are changing with different dynamics. For $\mathrm{LPS}_{\mathrm{PhOH}}$ and $\mathrm{LPS}_{\mathrm{H}_{2} \mathrm{O}}$ in concentrations between $0 \%$ and $0.4 \%$ the TEMPO probe (Fig. 3a) did not detect changes in the fluidity of the membrane-lipid interface, since no significant changes in parameters $F$ were recorded. Above this concentration the value of the $F$ parameter started to decrease, which may indicate an increase in the stiffness of the surface part of the membrane at the water-lipid interface. To better illustrate TEMPO $F$ parameter changes, Fig. 4 and Fig. 5 were included. Graphs show shape changes of recorded spectroscopic lines for selected $\mathrm{LPS}_{\mathrm{H}_{2} \mathrm{O}}$ concentrations. $0 \%$ indicates the reference spectrum (liposomes without LPS), $0.6 \%$ and $1.4 \%$ relate to liposomes doped with $\mathrm{LPS}_{\mathrm{H}_{2} \mathrm{O}}$

Figure 5 shows significant changes in peak height which are associated with increase of concentrations of $\mathrm{LPS}_{\mathrm{H}_{2} \mathrm{O}}$. Decrease of peak heights indicates that the spin probe migrates from a lipid environment (liposome membrane) to an aqueous environment (liposome surrounding area). This phenomenon might be interpreted as an increase of stiffness in the water-lipid interface $[21,22]$ caused by $\mathrm{LPS}_{\mathrm{H}_{2} \mathrm{O}}$. In liposomes doped by $\mathrm{LPS}_{\mathrm{PhOH}}$ those changes are smaller and occur in concentrations ranging

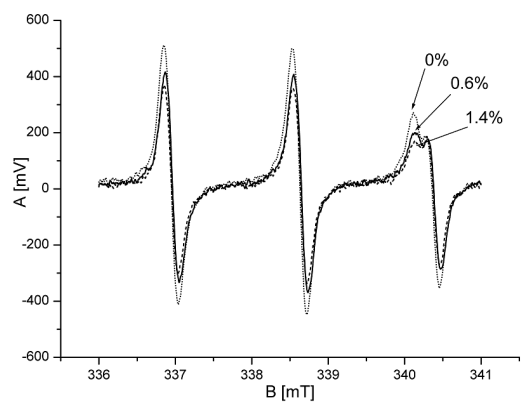

Fig. 4. Shape changes of spectroscopic lines recorded for TEMPO spin probe placed in DPPC liposome solution doped by $0.6 \%$ and $1.4 \% \mathrm{LPS}_{\mathrm{H}_{2} \mathrm{O}}$.

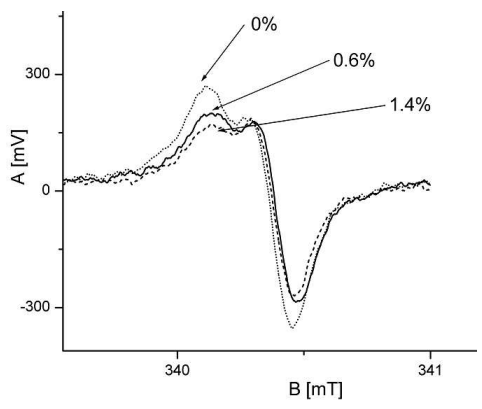

Fig. 5. The magnified part of Fig. 4 graph shows changes in the high-field line for TEMPO probe.

from $0.4 \%$ to $0.8 \%$. Above this concentration changes induced by $\mathrm{LPS}_{\mathrm{PhOH}}$ are stable. This may indicate a limited dissolution of the $\mathrm{LPS}_{\mathrm{PhOH}}$ in the liposome membranes at concentrations above $0.8 \%$. For the 16 -DOXYL spin probe located inside the lipid bilayer, minor changes in spectroscopic parameter $\tau$ were observed when liposomes were doped by LPS $_{\mathrm{PhOH}}$ in concentrations ranging from $0 \%$ to $0.4 \%$, while major changes were observed for $\mathrm{LPS}_{\mathrm{H}_{2} \mathrm{O}}$ in the same concentration range (Fig. 3b). The above observations for 16-DOXYL might indicate reduced fluidity of the central part of the lipid bilayer under the influence of $\mathrm{LPS}_{\mathrm{H}_{2} \mathrm{O}}$ and the lack of membrane reaction to $\mathrm{LPS}_{\mathrm{PhOH}}$. Some type of spectra 16-DOXYL probe is shown in Fig. 6 .

At concentrations above $0.4 \%$, a significant decrease

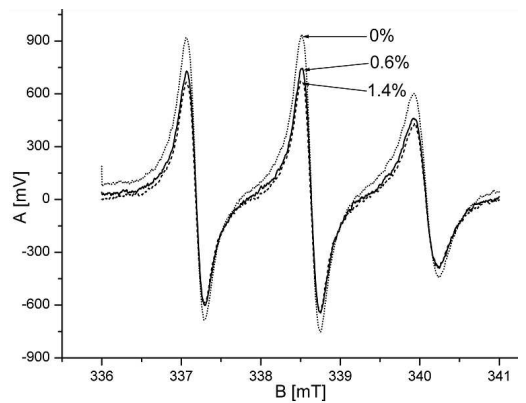

Fig. 6. Shape changes of spectroscopic lines recorded for 16-DOXYL spin probe placed in the central part of the DPPC liposome membranes doped by $0.6 \%$ and $1.4 \% \mathrm{LPS}_{\mathrm{H}_{2} \mathrm{O}}$. 
in the value of the $\tau$ parameter for membranes doped by $\mathrm{LPS}_{\mathrm{H}_{2} \mathrm{O}}$ and $\mathrm{LPS}_{\mathrm{PhOH}}$ was observed. A decrease in the value of the $\tau$ parameter indicates faster probe rotation which is related to viscosity reduction in the central part of the liposome lipid bilayer. Both LPS concentration above $0.8 \%$ induced a slowdown in the process of membrane liquefaction. Observed changes of the $\tau$ parameters for membranes doped by $\mathrm{LPS}_{\mathrm{H}_{2} \mathrm{O}}$ and $\mathrm{LPS}_{\mathrm{PhOH}}$ are very much alike which suggests a similar impact of these compounds on the fluidity of the central part of the liposome bilayer. While $\mathrm{LPS}_{\mathrm{PhOH}}$ appears to be more active, greater changes of the $\tau$ parameter were observed in concentrations ranging from $0.6 \%$ to $1.4 \%$. This is probably due to better penetration (better dissolution) in the hydrophobic environment. Results obtained for the TEMPO probe confirm the above observations. In the case of the $F$ parameter, the strongest impact on this parameter was recorded for $\mathrm{LPS}_{\mathrm{H}_{2} \mathrm{O}}$ which is more soluble in the hydrophilic environment. Such an environment in the tested membranes is a layer of polar head groups, to which the TEMPO probe is particularly sensitive [23-25].

\section{Conclusion}

Both $\mathrm{LPS}_{\mathrm{H}_{2} \mathrm{O}}$ and $\mathrm{LPS}_{\mathrm{PhOH}}$ for concentrations ranging from $0.4 \%$ to $0.8 \%$ (molar ratio to lecithin) significantly changed the fluidity of liposome membranes. $\mathrm{LPS}_{\mathrm{H}_{2} \mathrm{O}}$ exhibited a higher impact on the membrane of the polar head groups area while LPS $_{\mathrm{PhOH}}$ influenced the central part of the lipid bilayer. This peculiarity was probably affiliated with the different polarity of LPS molecules $\left(\mathrm{LPS}_{\mathrm{H}_{2} \mathrm{O}}\right.$ and $\left.\mathrm{LPS}_{\mathrm{PhOH}}\right)$. LPS of P. shigelloides strain CNCTC 144/92 (serotype O74:H5) which is a S-LPS obtained with the hot phenol/water method showed unexpectedly higher yields from the phenol phase $\left(\mathrm{LPS}_{\mathrm{PhOH}}\right)$ [9]. According to the authors the SDS-PAGE analysis of $\mathrm{LPS}_{\mathrm{H}_{2} \mathrm{O}}$ and $\mathrm{LPS}_{\mathrm{PhOH}}$ revealed that $\mathrm{LPS}_{\mathrm{PhOH}}$ consists of a higher number of oligosaccharide repeating units in the O-PS region which is typical for S-LPS. The lipid $A$ which is the most hydrophobic region of LPS was also analyzed separately for $\mathrm{LPS}_{\mathrm{H}_{2} \mathrm{O}}$ and $\mathrm{LPS}_{\mathrm{PhOH}}$. There were no differences in the structures. The heterogeneity of lipid A from $\mathrm{LPS}_{\mathrm{H}_{2} \mathrm{O}}$ and $\mathrm{LPS}_{\mathrm{PhOH}}$ included different acylation patterns [26]. In summary, one may assume that heterogeneity in the lipid $A$ structure and different numbers of oligosaccharide repeating units in the O-PS of $\mathrm{LPS}_{\mathrm{H}_{2} \mathrm{O}}$ and $\mathrm{LPS}_{\mathrm{PhOH}}$ might be responsible for the different behavior in the liposome membranes when analyzed by EPR spin probe technique.

\section{Acknowledgments}

We wish to thank Prof. Czesław Lugowski for providing LPS of $P$. shigelloides strain CNCTC 144/92 (serotype O74:H5) extracted from phenol and water phase and Aleksandra Man-Kupisińska for substantial assistance on LPS, from the Department of Immunochemistry, Ludwik Hirszfeld Institute of Immunology and Experimental Therapy, PAS.

\section{References}

[1] D. Man, R. Słota, M.A. Broda, G. Mele, J. Li, J. Biol. Inorg. Chem. 16, 173 (2011).

[2] D. Man, I. Pisarek, M. Braczkowski, Nukleonika 58, 439 (2013).

[3] D. Man, I. Pisarek, M. Braczkowski, B. Pytel, R. Olchawa, J. Lipos. Res. 24, 106 (2014).

[4] B. Pytel, A. Filipiak, I. Pisarek, R. Olchawa, D. Man, Nukleonika 60, 455 (2015).

[5] R. Olchawa, D. Man, B. Pytel, Nukleonika 60, 395 (2015).

[6] D. Man, J. Liposome Res. 18, 225 (2008).

[7] R. Wałęsa, D. Man, G. Engel, D. Siodłak, T. Kupka, T. Ptak, M.A. Broda, Chem. Biodivers. 12, 1007 (2015).

[8] J.J. Farmer III, M.J. Arduino, F. Hickman-Brenner, in: The Prokaryotes, Vol. 6, Eds. M. Dworkin, S. Falkow, E. Rosenberg, K.-H. Schleifer, E. Stackebrandt, Springer, New York 2006, p. 564.

[9] S. Yamada, S. Matsushita, S. Dejsirilert, Y. Kudoh, Epidemiol. Infect. 119, 121 (1997).

[10] H. Rautelin, A. Sivonen, A. Kuikka, O.V. Renkonen, V. Valtonen, T.U. Kosunen, Scand. J. Infect. Dis. 27, 495 (1995).

[11] A.C.W. Lee, K.Y. Yuen, S.Y. Ha, D.C.K. Chiu, Y.L. Lau, Pediatr. Hematol. Oncol. 13, 265 (1996).

[12] R.B. Clark, G.R. Westby, H. Spector, R.R. Soricelli, C.L. Young, J. Infect. 23, 89 (1991).

[13] K. Fujita, M. Shirai, T. Ishioka, F. Kakuya, Acta Paediatr. Jpn. 36, 450 (1994).

[14] B. Beutler, E.Th. Rietschel, Nat. Rev. Immunol. 3, 169 (2003).

[15] O. Westphal, K. Jann, in: Methods in Carbohydrate Chemistry, Vol. 5, Ed. R.L. Whistler, Academic Press, London 1965, p. 83.

[16] T. Niedziela, S. Dag, J. Lukasiewicz, M. Dzieciatkowska, W. Jachymek, C. Lugowski, L. Kenne, Biochemistry 45, 10422 (2006).

[17] S.R. Haseley, O. Holst, H. Brade, Eur. J. Biochem. 247, 815 (1997).

[18] S.R. Haseley, O. Holst, H. Brade, Eur. J. Biochem. 251, 189 (1998).

[19] M. Gao, W. D'Haeze, R. De Rycke, B. Wolucka, M. Holsters, Mol. Plant-Microbe Interact. 14, 857 (2001).

[20] M. Caroff, D.R. Bundle, M.B. Perry, Eur. J. Biochem. 139, 195 (1984).

[21] E.J. Shimshick, H.M. McConnell, Biochemistry 12, 2351 (1973).

[22] M.A. Hemminga, Chem. Phys. Lipids 32, 323 (1983).

[23] D. Man, R. Olchawa, K. Kubica, J. Lipos. Res. 20, 211 (2010).

[24] D. Man, R. Olchawa, J. Lipos. Res. 23, 327 (2013).

[25] D. Man, R Olchawa, Eur. Biophys. J. 46, 325 (2016).

[26] J. Lukasiewicz, M. Dzieciatkowska, T. Niedziela, W. Jachymek, A. Augustyniuk, L. Kenne, C. Lugowski, Biochemistry 45, 10434 (2006). 